\title{
A Meta-Analysis of the Association Between the I148M Variant of Patatin- Like Phospholipase Domain Containing 3 Gene and the Presence of Chronic Hepatitis C
}

\author{
Haiying Zhang, ${ }^{1,2,}$ Li Xue, ${ }^{1,2}$ Lizhen Chen, ${ }^{3}$ Shunshun Jiang, ${ }^{1,2}$ Yongning Xin, ${ }^{4}$ and Shiying \\ Xuan $^{4, "}$ \\ ${ }^{1}$ Department of Gastroenterology, The Second Affiliated Hospital of Medical College, Qingdao University, Qingdao, China \\ ${ }_{3}^{2}$ Department of Gastroenterology, Qingdao Central Hospital, Qingdao, China \\ ${ }^{3}$ Medical College, Qingdao University, Qingdao, China \\ ${ }^{4}$ Department of Gastroenterology, Qingdao Municipal Hospital, Qingdao, China \\ ${ }^{*}$ Corresponding Authors: Haiying Zhang, Department of Gastroenterology, The Second Affiliated Hospital of Medical College, Qingdao University, Qingdao, China. Tel: \\ +86-53284963627, Fax: +86-53288905293, E-mail: zhanghaiying920@163.com; Shiying Xuan, Department of Gastroenterology, Qingdao Municipal Hospital, Qingdao, \\ China. Tel: +86-53288905289, Fax: +86-53288905293, E-mail: dxyxyn@163.com
}

Received 2015 August 1; Revised 2015 September 20; Accepted 2015 October 2.

\begin{abstract}
Context: The objective of the current study was to evaluate the association between the I148M variant of patatin-like phospholipase domain-containing protein 3 (PNPLA3) and the presence of Chronic Hepatitis $\mathrm{C}$ (CHC) across different populations.

Evidence Acquisition: This study was a meta-analysis of all relevant researches published in the literature from year 2000 to 2015 . The odds ratios (ORs) of PNPLA3 allele distributions in CHC patients were analyzed and compared with healthy controls. The meta-analysis Revman 5.2 software was applied for investigating heterogeneity among individual studies and for summarizing all the studies. The metaanalysis was carried out according to the Cochrane Reviewers' Handbook recommendations. A total of 120 clinical trials or reports were retrieved, yet only five trials met the study selection criteria.

Results: Five hospital-based case-control studies were included in the final analysis. The overall frequency of PNPLA3 gene polymorphisms was 20.4\% (205/1005) in CHC and 10.23\%(53/518) in controls. The summary odds ratio for the association of gene polymorphisms of PNPLA3 with the risk for $\mathrm{CHC}$ was determined as 2.20 (95\% CI: 1.56-3.11) and was statistically significant $(\mathrm{P}<0.05)$.

Conclusions: The current meta-analysis showed an association between frequency of GG genotype of PNPLA3 and the risk of development of $\mathrm{CHC}$ in various populations throughout the world.
\end{abstract}

Keywords: PNPLA3, Polymorphisms, CHC

\section{Context}

Hepatitis C virus (HCV) is a common hepatotropic RNA virus, which has been estimated to infect about 170 million people worldwide (1). The HCV infection progresses to chronicity in nearly $80 \%$ of cases, and chronic hepatitis $\mathrm{C}(\mathrm{CHC})$ infection is associated with the development of cirrhosis, end-stage liver disease, hepatocellular carcinoma, liver transplantation and associated complications in Western countries (2).

Differences in CHC prevalence, clinical profile and histological severity between different ethnic groups suggested a genetic contribution (3). Thus, in earlier candidate gene studies, several single nucleotide polymorphisms (SNPs) within host genes and gene regions coding for the human leukocyte system, keratin, or coagulation factors were shown to be associated with progression of $\mathrm{HCV}$ - induced liver fibrosis (4). Among these, an independent genome-wide association study that identified a non-synonymous sequence variation (rs738409 C > G), encoding an isoleucine-to-methionine substitution at position 148 in the adiponutrin/patatin-like phospholipase domaincontaining 3 (PNPLA3) gene, has recently attracted much interest (5).

The $\mathrm{G}$ allele of the rs738409 variant leads to triglyceride (TG) accumulation in hepatocytes. Steatosis can promote inflammatory mediators and oxidative stress and has been shown to favor hepatocyte apoptosis in CHC (6). Steatosis is also closely associated with metabolic syndrome and insulin resistance, a well-established cause of fibrosis progression in hepatitis C (7). The association of steatosis and $\mathrm{CHC}$ has been well described, and shown to

Copyright ( 2015, Kowsar Corp. This is an open-access article distributed under the terms of the Creative Commons Attribution-NonCommercial 4.0 International License (http://creativecommons.org/licenses/by-nc/4.0/) which permits copy and redistribute the material just in noncommercial usages, provided the original work is properly cited. 
occur in up to $66 \%$ of cases $(6,8)$. Steatosis accelerates the progression of $\mathrm{CHC}$ and is independently associated with stage III/IV hepatic fibrosis (8).

Single nucleotide polymorphisms (SNPs) have also been reported to be associated not only with elevated liver enzymes in healthy subjects, but also with disease severity, portal inflammation, lobular inflammation, steatosis, fibrosis, and hepatocellular carcinoma (9). More recently, the $G$ allele of the rs738409 variant has been reported to be associated with the occurrence of CHC (10). In view of the uncertain association of the I148M variant of PNPLA3 in CHC, we conducted a metaanalysis to comprehensively assess the overall performance of the I148M variant of PNPLA3 for the presence of CHC, and to analyze the heterogeneity between available studies before its wide application in clinical practice. The aim of this study was to evaluate the association of the I148M variant of PNPLA3 and the presence of $\mathrm{CHC}$ across various populations.

\section{Evidence Acquisition}

\subsection{Search Strategy}

The objective of our search was to identify published genetic association studies evaluating the gene polymorphisms of PNPLA3 and CHC in humans, written in all languages, between years 2000 to June 2015. This aim was achieved by performing a meta-analysis. An electronic search was completed using PubMed, EMBASE, the Cochrane Library, Scopus, Index Copernicus, DOAJ, EBSCOCINAHL, and the China National Knowledge Infrastructure. The search term was based on combinations of the following key words; "patatin like phospholipase domain containing 3 or PNPLA3 or adiponutrin, and chronic hepatitis C or CHC or HCV", and was not restricted by period. A full manual search from the bibliographies of selected papers was also performed. In addition, authors of gray literatures and studies containing relevant information, yet lacking the data necessary for this analysis, were contacted directly. Unpublished data were also accepted if an abstract was available and further information was obtained from the authors.

\subsection{Inclusion and Exclusion Criteria}

In this meta-analysis, two independent investigators reviewed and searched the following types of studies: 1) an independent case-control study; 2) studies with similar purpose and statistical methods; 3) studies providing enough information to calculate an Odds Ratio (OR); 4) stratified outcomes according to the PNPLA3 genotype; 5) studies with diagnosis of $\mathrm{CHC}$ based on the detection of both HCV antibodies and HCV RNA for at least six months in the presence of signs of chronic hepatitis, and elevated aminotransferases or histology; and 6) studies with genotyping performed with a vali- dated molecular method. Because the variation seemed to follow an undefined model of inheritance in some of the outcomes, to avoid choosing any a priori model, it was decided to compare extreme genotypes, namely, homozygous CC (148 I/I) versus homozygous GG (148 M/M) reported by a previous study (11). In addition, in order to address which genetic model best explains the effect of rs738409 SNP on the susceptibility to develop CHC, an evaluation of risks associated with heterozygosity of the variant (heterozygous CG versus homozygous CC, the reference group) was also included. For each phenotype, the association results sub-grouped by ethnicity were evaluated.

The exclusion criteria were: 1) incomplete raw data; 2) non-human data; 3 ) repetitive reports (if more than one version of the same study was retrieved, only the most recent was used); 4) other potential causes of liver disease (including alcohol, non-alcoholic fatty liver disease (NAFLD), other viral infections such as hepatitis B virus and human immunodeficiency virus, previous treatment, and the use of drugs); and 5) materials and methods not well-described or reliable.

Although assessment of the study quality is considered important for systematic reviews and meta-analyses, scoring methods have been considered problematic (12), and may not accurately assess the quality measures of interest (13). Therefore, the reliability of patient selection, molecular typing method, HCV genotyping, and statistical analysis methods were used as quality variables. The investigators assessed the risk of bias in the included studies using a risk-of-bias tool. Any disagreement was resolved through discussion among the authors until a consensus was reached. The following studies were considered as low-bias risk trials, those that had an adequate handling of incomplete outcome data, were free of selective reporting, included an adequate intervention description, had appropriate criteria for participant recruitment and included an adequate outcome explanation. Studies with one or more unclear or inadequate quality component were considered highbias risk trials.

\subsection{Data Extraction}

Two investigators (Zhang HY and Xin YN) independently evaluated study eligibility, graded quality, and extracted outcome data. Disagreements were resolved by consensus. For each study, the following information were recorded: demographic information (age, sex and ethnicity), authors, publication year, journal, publication type and language, PNPLA3 genotyping method, allele genotype, number of cases and controls, definitions used for $\mathrm{CHC}$, and $\mathrm{CHC}$ sample description. Furthermore, the healthy group was defined as the control group to minimize confounding factors. The main features of the trials included in the meta-analysis are shown in Table 1. 
Table 1. Characteristics of Studies on the Association Between Gene Polymorphisms of Patatin-Like Phospholipase Domain-Containing 3 and Chronic Hepatitis $C^{\mathrm{a}}$

\begin{tabular}{|c|c|c|c|c|c|c|c|c|c|c|}
\hline \multirow[t]{2}{*}{ First Author Year } & \multirow[t]{2}{*}{ Ref. } & \multirow{2}{*}{$\begin{array}{c}\text { Study/Center } \\
\text { Description }\end{array}$} & \multirow{2}{*}{$\begin{array}{c}\text { Population } \\
\text { Ethnicity Country }\end{array}$} & \multicolumn{2}{|c|}{$\mathrm{CHC}$} & \multicolumn{2}{|c|}{ Controls } & \multirow{2}{*}{$\begin{array}{c}\text { Liver } \\
\text { Biopsy }\end{array}$} & \multirow{2}{*}{$\begin{array}{c}\text { No. of Genotype } \\
1 / 2 / 3 \text { in CHC }\end{array}$} & \multirow{2}{*}{$\begin{array}{l}\text { QUADAS } \\
\text { Score }\end{array}$} \\
\hline & & & & No. $(M / F)$ & Age, $y$ & No. $(M / F)$ & Age, $y$ & & & \\
\hline Nischalke et al. 2011 & (14) & $\begin{array}{l}\text { prospective, } \\
\text { one center }\end{array}$ & $\begin{array}{c}\text { Bonn and Berlin } \\
\text { Caucasians }\end{array}$ & $162(92 / 70)$ & 56 & $190(106 / 84)$ & 40.5 & $\mathrm{Y}$ & NA & 12 \\
\hline Valenti et al. 2011 & (15) & $\begin{array}{l}\text { prospective, } \\
\text { multicenter }\end{array}$ & Italian Caucasians & $819(357 / 462)$ & 57.35 & $179(141 / 38)$ & 48.4 & $\mathrm{Y}$ & $468 / 222 / 55$ & 13 \\
\hline Miyashita et al. 2012 & $(16)$ & $\begin{array}{l}\text { prospective, } \\
\text { multicenter }\end{array}$ & Japanese & $220(88 / 132)$ & $55.0 \pm 0.58$ & $85(-/-)$ & ND & $\mathrm{Y}$ & $194 / 21 / N A$ & 11 \\
\hline Valenti et al. 2012 & (17) & $\begin{array}{l}\text { prospective, } \\
\text { one center }\end{array}$ & Italian Caucasians & $261(151 / 110)$ & $58.1 \pm 14$ & $257(202 / 55)$ & $48 \pm 12$ & $\mathrm{Y}$ & NA & 13 \\
\hline Ezzikouri et al. 2014 & (18) & $\begin{array}{c}\text { prospective, } \\
\text { one center }\end{array}$ & $\begin{array}{c}\text { Moroccan Berber } \\
\text { and Arabic }\end{array}$ & $230(104 / 126)$ & $63.63 \pm 12.28$ & $132(62 / 70)$ & $56.27 \pm 10.64$ & $\mathrm{NA}$ & $162 / 68 / \mathrm{NA}$ & 11 \\
\hline
\end{tabular}

a Abbreviations: PNPLA3: Patatin-Like Phospholipase Domain-Containing 3; CHC: Chronic Hepatitis C; NA: Not Available.

\subsection{Statistical Analysis}

The authors were not blinded to the names of the studies' authors, journals and results. Any disagreements were resolved through discussion among the authors until consensus was reached (Kappa coefficient: 78\%). Homogeneity was evaluated by Cochran's $Q$ test $(\alpha=0.05)$ and the I2 statistic. If the results of the test had no significant heterogeneity, the Mantel-Haenszel fixed-effect model (Peto method) was used for the combined data. If the results of the test had significant heterogeneity, the Dersimonian-Laird random-effects model (DL method) was used for the combination of data (19). An I2 value of $0 \%$ indicated no heterogeneity, and if $\mathrm{I} 2$ was $<50 \%$, the test was considered uniform with no statistically significant heterogeneity, while larger values showed an increasing heterogeneity. A pooled OR was presented as a standard plot with $95 \%$ confidence intervals (CIs). In the absence of heterogeneity, the two methods provided identical results. As a measure of association between CHC and PNPLA3 alleles, ORs with 95\% CIs, stratified by gene subtypes of patients and controls in a study, were combined. Funnel plots were used to evaluate publication bias (20). A sensitivity analysis was performed to assess the stability of the results by sequential omission of individual studies. All presented P Values were two-tailed. The analyses were performed using Revman 5.2, provided by the Cochrane Collaboration Internet.

\section{Results}

A total of 120 studies were retrieved based on the described search strategies. Eight eligible studies were identified for evaluation. Ultimately, three studies were excluded for having insufficient data. Thus, our final dataset for the meta-analysis (Figure 1) included five studies (14-18). The main features of the studies, included in the meta-analysis, are shown in Table 1. The genotype distributions of rs738409 polymorphism of PNPLA3 among included studies are shown in Table 2. A total of 2535 subjects were included (1692 patients and 843 healthy controls). Two of these studies were conducted in Italy $(15,17)$, and one in Bonn and Berlin (14), Japan (16) and Morocco (18). All of the five studies were hospital-based case-control studies (14-18). Information about liver biopsies was available for four studies (14-17). Hepatitis C Virus genotyping was performed using the TaqMan assay in three studies $(15,16,18)$. All studies scored well in terms of adequate descriptions of selection criteria and availability of clinical data.

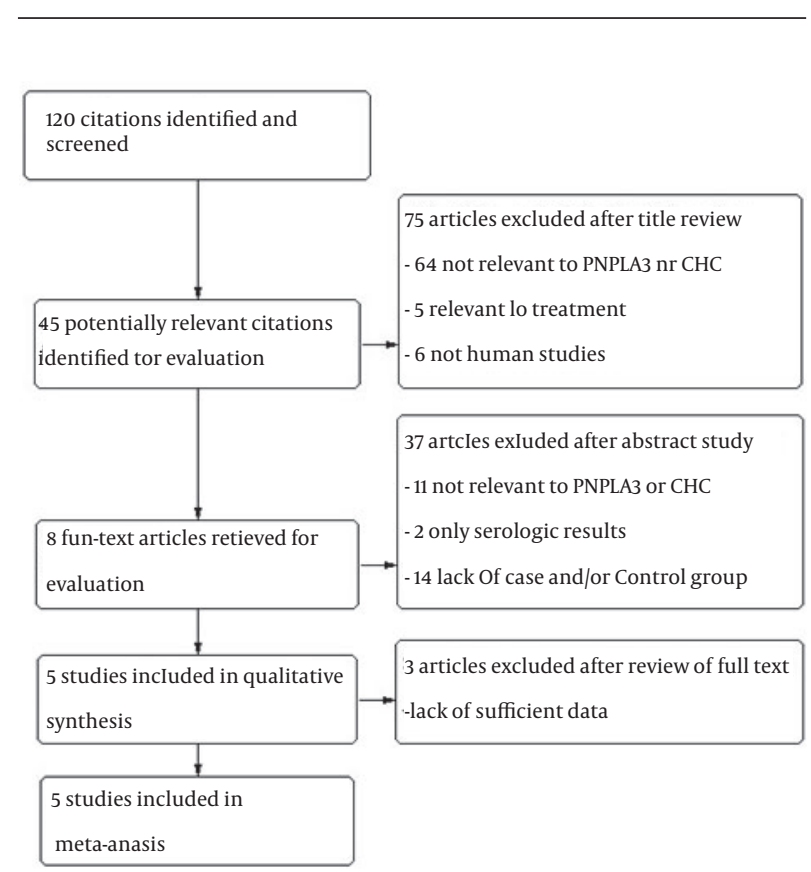

Figure 1. Flow Diagram of the Identification Procedure of the Studies 
The comparison between cases and controls showed that $\mathrm{CHC}$ was significantly associated with the GG genotype. In the meta-analysis, the overall frequency of the GG genotype distribution was $20.4 \%$ (205/1005) in CHC, and $10.23 \%$ (53/518) in controls. The heterogeneity test indicated that the variation of trial-specific ORs was not statistically significant $\left(\chi^{2}=4.22, \mathrm{P}=0.38\right)$; the fixed-effect method was used to combine the results. The combined OR was 2.20 (95\% CI: 1.56 - 3.11) and was statistically significant $(\mathrm{P}<0.00001)$. In the sensitivity analysis, the exclusion of individual studies did not change this significant result. Statistics calculated for studies assessing the association between gene polymorphisms of PNPLA3 and $\mathrm{CHC}$ when comparing homozygous GG and homozygous CC, are shown in the forest plot (Figure 2).

The analysis of the heterozygosity for the variant showed that $\mathrm{CHC}$ was significantly associated with the rs738409 $\mathrm{G}$ allele when the reference heterozygous CG was compared with homozygous CC, again suggesting an additive genetic effect (details in Figure 3). The heterogeneity test indicated that the variation of trial-specific ORs was not statistically significant $\left(\chi^{2}=5.86, P=0.21\right)$; the fixed-effect method was used to combine the results. The combined OR was 1.26 (95\% CI: 1.04 - 1.51) and was statistically significant $(\mathrm{P}=0.02)$. In the sensitivity analysis, the exclusion of individual studies did not change this significant result. The current meta-analysis revealed that there was an association between gene polymorphisms of PNPLA3 and CHC. Gene polymorphisms of PNPLA3 were a risk factor for the presence of CHC. These analyses were based on data from studies irrespective of population ethnicity. Funnel plots to detect publication bias of studies on PNPLA3 tended towards an asymmetrical shape (Figures 4 and 5), suggesting that publication bias might have affected the findings of our meta-analysis.

Specific ORs was not statistically significant $\left(\chi^{2}=3.42, \mathrm{P}\right.$ $=0.18$ ) when the GG genotype was compared with the CC genotype. The fixed-effect method was used to combine the results. The combined OR was 2.51 (95\% CI: 1.60 - 3.95), which was statistically significant $(\mathrm{P}<0.0001)$ (Figure 6$)$. The same result was also found when the reference genotype CG was compared with the CC genotype (details in Figure 7). The heterogeneity test indicated that the variation of trial-specific ORs was not statistically significant $\left(\chi^{2}=1.17, \mathrm{P}=0.56\right)$; the fixed-effect method was used to combine the results. The combined OR was 1.33 (95\% CI: $1.07-1.65)$ and was statistically significant $(P=0.01)$. The subgroup analyses of ethnicity revealed a remarkable association between gene polymorphisms of PNPLA3 and $\mathrm{CHC}$ in Caucasians.

Table 2. Genotype Distribution of Patatin-Like Phospholipase Domain-Containing 3 Amongst the Reviewed Studies ${ }^{\mathrm{a}}$

\begin{tabular}{|c|c|c|c|c|c|c|c|c|}
\hline \multirow[t]{2}{*}{ First Author, Y } & \multirow[t]{2}{*}{ Ref. } & \multirow[t]{2}{*}{ Number of Patients } & \multicolumn{3}{|c|}{$\mathrm{CHC}$} & \multicolumn{3}{|c|}{ Controls } \\
\hline & & & $\mathrm{CC}$ & CG & GG & CC & CG & GG \\
\hline Nischalke et al. 2011 & (14) & 352 & 85 & 64 & 13 & 112 & 69 & 9 \\
\hline Valenti et al. 2011 & $(15)$ & 998 & 424 & 310 & 85 & 118 & 56 & 5 \\
\hline Miyashita et al. 2012 & (16) & 305 & 68 & 104 & 48 & 23 & 51 & 11 \\
\hline Valenti et al. 2012 & (17) & 518 & 133 & 103 & 25 & 146 & 95 & 16 \\
\hline Ezzikouri et al.2014 & (18) & 362 & 90 & 106 & 34 & 66 & 54 & 12 \\
\hline
\end{tabular}

a Abbreviations: PNPLA3: patatin-like phospholipase domain-containing 3; CHC: chronic hepatitis C; CC: homozygous CC; GG: homozygous GG.

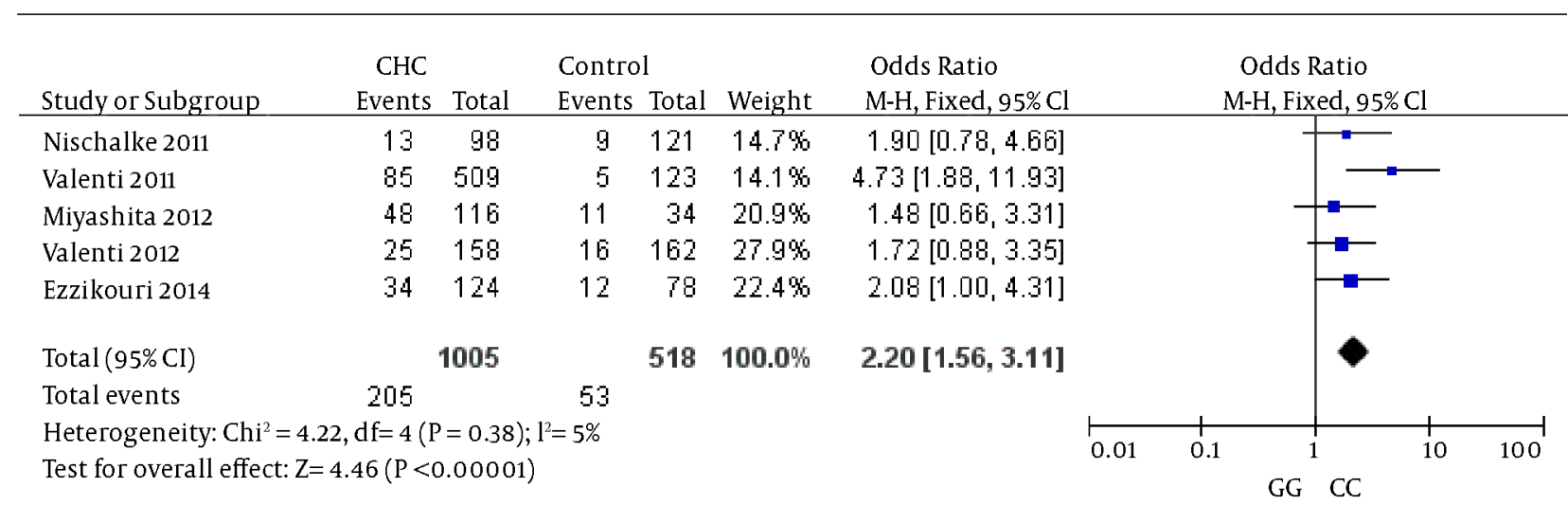

Figure 2. Meta-Analysis Forest Plot of Included Studies on the Association Between Gene Polymorphisms of Patatin-Like Phospholipase Domain-Containing 3 and Chronic Hepatitis C (CG Vs. CC Genotypes) 
Zhang Het al.

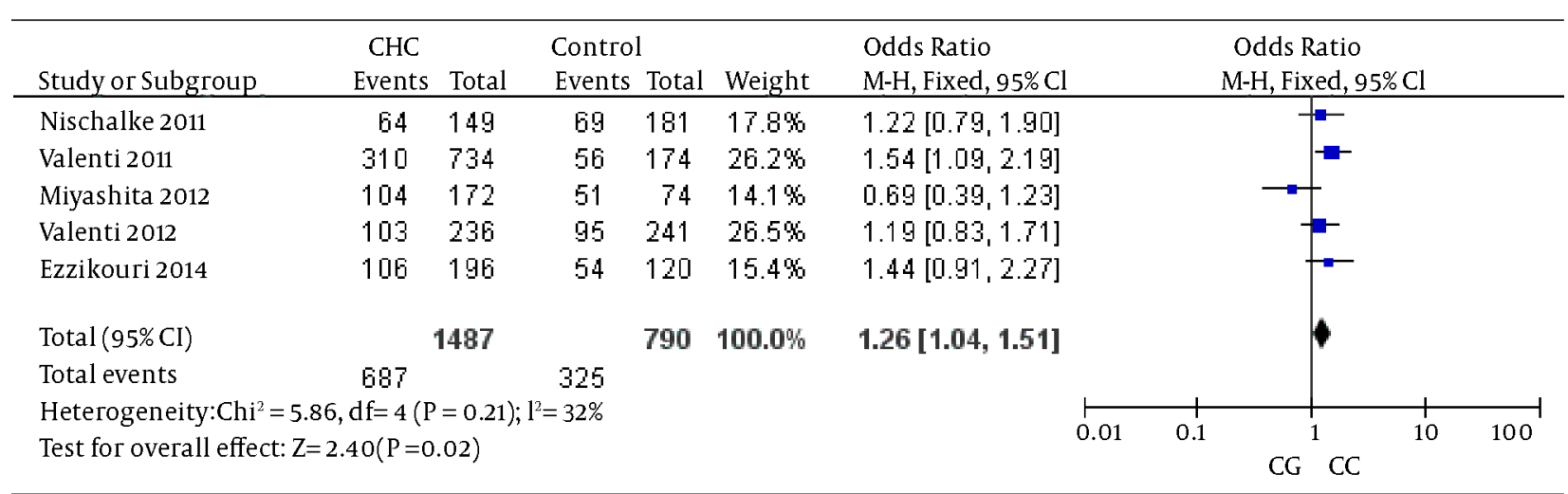

Figure 3. Meta-Analysis Forest Plot of Included Studies on the Association Between Gene Polymorphisms of Patatin-Like Phospholipase Domain-Containing 3 and Chronic Hepatitis C (CG Vs. CC Genotypes)

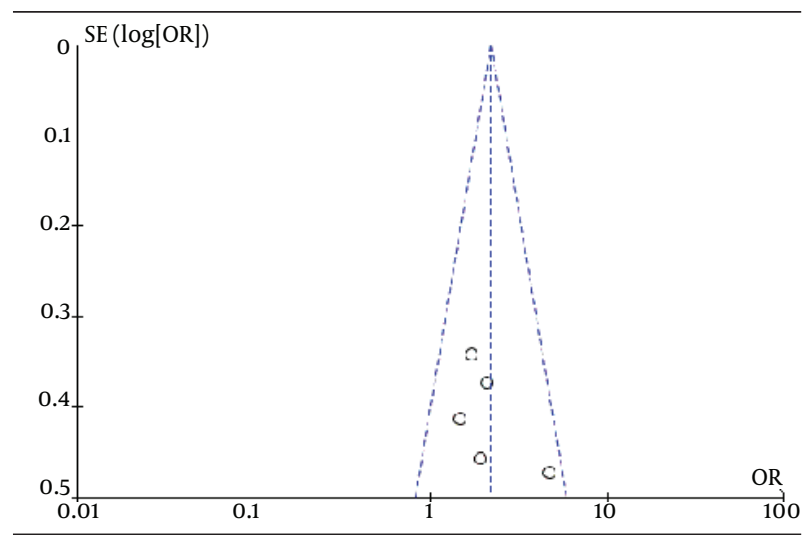

Figure 4. Meta-Analysis Funnel Plot of Included Studies on the Association Between Gene Polymorphisms of Patatin-Like Phospholipase Domain-Containing 3 and Chronic Hepatitis C (GG Vs. CC Genotypes)

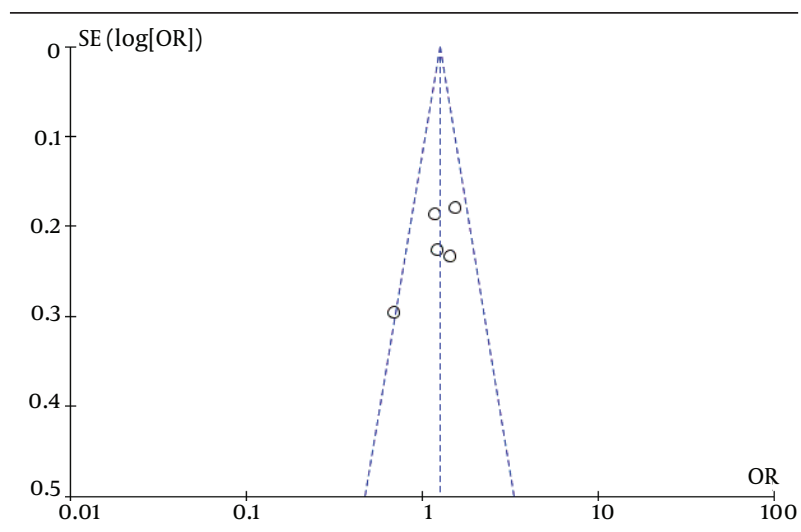

Figure 5. Meta-Analysis Funnel Plot of Included Studies on the Association Between Gene Polymorphisms of Patatin-Like Phospholipase Domain-Containing 3 and Chronic Hepatitis C (CG Vs. CC Genotypes)

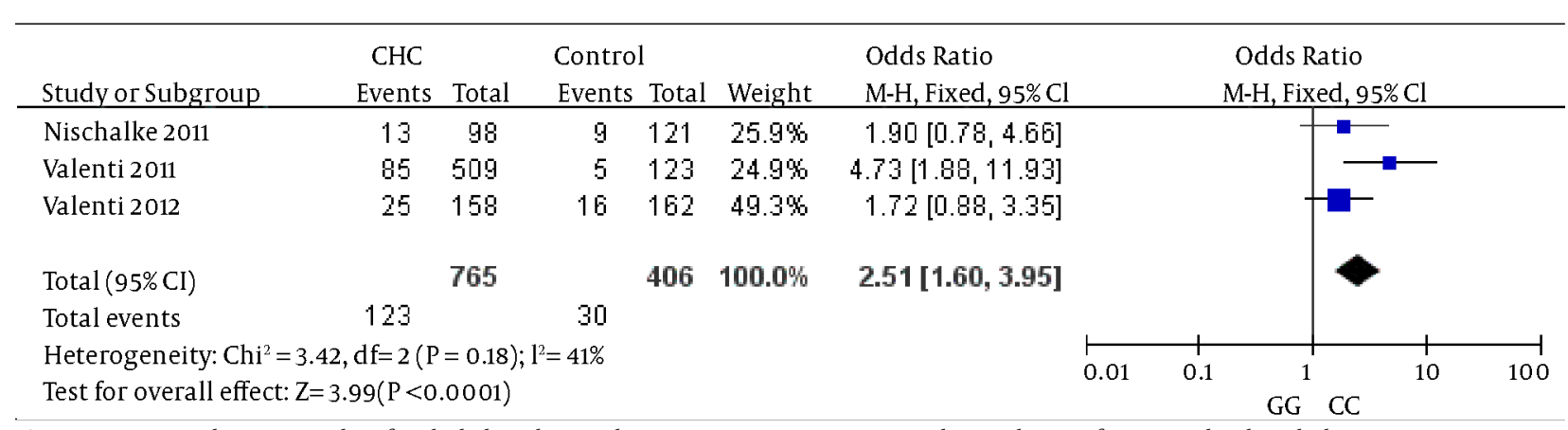

Figure 6. Meta-Analysis Forest Plot of Included Studies on the Association Between Gene Polymorphisms of Patatin-Like Phospholipase Domain-Containing 3 and Chronic Hepatitis C in Caucasians (GG Vs. CC Genotypes)

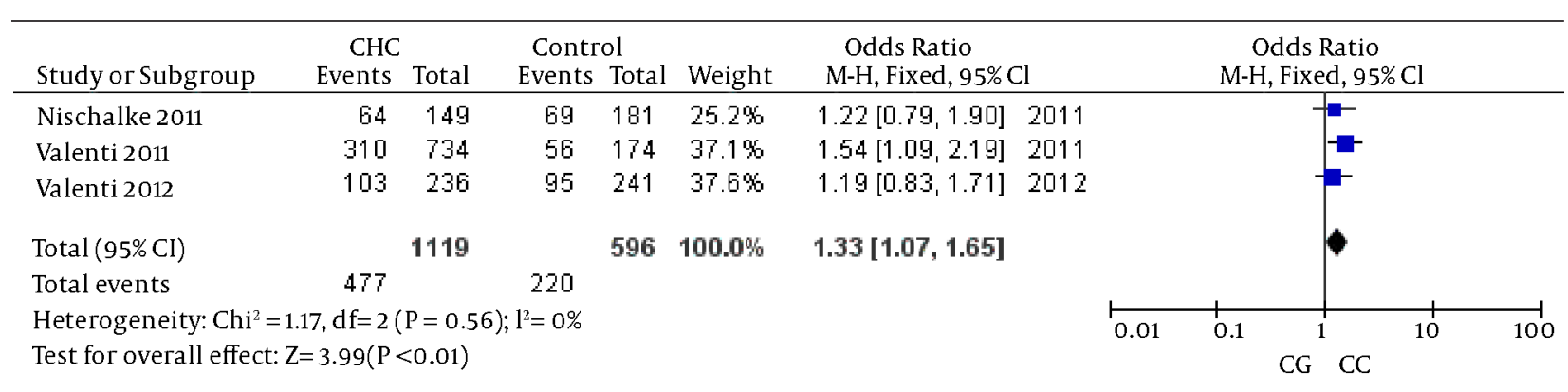

Figure 7. Meta-Analysis Forest Plot of Included Studies on the Association Between Gene Polymorphisms of Patatin-Like Phospholipase Domain-Containing 3 and Chronic Hepatitis C in Caucasians (CG Vs. CC Genotypes) 
Patatin-like phospholipase domain-containing 3, also called adiponutrin, encodes a 481-amino acid protein with a molecular mass of approximately $53 \mathrm{kDa}$ that, in humans, is mainly expressed in intracellular membrane fractions in hepatocytes (21). Furthermore, PNPLA3 is induced in the liver after feeding and during insulin resistance by the master regulator of lipogenesis steroid regulatory element binding protein-1c (22). Wild-type (148I) PNPLA3 has lipolytic activity towards TG (21). The 148M mutation determines a critical amino acid substitution next to the catalytic domain, likely reducing access of substrates and the PNPLA3 enzymatic activity towards glycerolipids, thereby leading to the development of macrovesicular steatosis (23). The presence of steatosis has been associated with more aggressive histological features, faster progression of fibrosis, and poorer response to therapy. As for the mechanisms of lipolytic activity of PNPLA3, there is another study has reported on lipogenic function associated with the $148 \mathrm{M}$ variant, which would acquire the ability to synthesize phosphatidic acid from lysophosphatidic acid (24). The functional consequences of the I148M polymorphism is therefore still powerfully debated, and it may be hypothesized that PNPLA3 has additional physiological substrates. Human studies have also suggested a possible direct or indirect influence of PNPLA3 genotype on adipose.

In contrast to studies on both nonalcoholic fatty liver disease (NAFLD) and alcoholic liver disease (ALD), liver damage (steatosis and fibrosis) related to PNPLA3 in CHC appears to primarily involve the homozygote $\mathrm{G}$ allele carriers, as reported by previous studies (10). Among the first to demonstrate the impact of the PNPLA polymorphism in patients with HCV infection were the studies of Valenti et al. (15) and Trepo et al. (25). They analyzed large cohorts of HCV patients in Europe and almost simultaneously reported the G allele of rs738409 SNP to be associated not only with the presence of histologically-determined liver steatosis, but also with the presence of cirrhosis and accelerated fibrosis progression. Corradini et al. further expanded these findings by reporting on an increased rate of hepatocellular carcinoma development in patients with the GG genotype of rs738409 in a cohort of $222 \mathrm{HCV}$ patients (26). Indeed, by multivariate analysis, the GG genotype was found to be an independent factor associated with HCC development carrying a 2.23 OR. Taken together, these data rather unequivocally demonstrate a correlation between the G allele of the PNPLA3 SNP and a worse prognosis of $\mathrm{CHC}$.

There are some points about the PNPLA3 SNP genotype that might explain its association with the progression of CHC. First, steatosis is known to negatively impact the natural history of HCV infection as it accelerates progression to cirrhosis (27). Second, liver steatosis has been shown to be a negative moderator of treatment outcome to interferon-based therapies. Patients with fatty liver had low sustained virological response rates across all HCV genotypes (28). Steatosis can promote inflammatory mediators and oxidative stress and has been shown to favor hepatocyte apoptosis in $\mathrm{CHC}$ (29). In addition to the effects on TG, the PNPLA3 genotype inhibits inflammation and fibrogenesis in the presence of severe liver fibrosis.

Adiponectin resistance may modify the interaction between genotype and environmental factors, which may increase the risk of developing CHC. An increased risk for addictive behavior, anemias requiring transfusions, percutaneous contact with infected blood, injection drug use, and tissue donations account for most HCV infections worldwide, which could influence the risk of having CHC. Whether the PNPLA3 genotype affects these factors is not known, and the exact mechanism behind this association remains poorly understood. Furthermore, PNPLA3 does not appear to have any direct association with $\mathrm{HCV}$, as there is no known effect on viral load or treatment response. It is possible that this association is simply related to confounders, such as increased fibrosis from concomitant alcohol abuse or metabolic syndrome, among patients with the PNPLA3 GG genotype.

In subgroup analyses of ethnicity, the heterogeneity test indicated that the variation of trial-

The results of our meta-analysis showed that the $G$ allele of the PNPLA3 rs738409 G > C SNP was associated with the presence of CHC. Individuals with the GG genotype had approximately a two-fold higher risk of having $\mathrm{CHC}$ when compared to individuals with the CC genotype. The GC heterozygous genotype was also associated with a smaller, but still significant risk. To our knowledge, this is the first published meta-analysis to comprehensively investigate the association between gene polymorphisms of PNPLA3 and CHC. In the current study, only publications in the English or Chinese language were analyzed. 'Meta-analytical' research, on 29 meta-analyses, investigating language bias has provided evidence that the $\mathrm{OR}$ estimated in meta-analyses from non-English publications were on average 0.8 -fold ( $95 \% \mathrm{CI}, 0.7-1.0)$ the OR estimates from English-written publications (30). For this reason, even if non-English publications had not been searched, this might have introduced only a small bias in the overall findings. Therefore, our language methodology is unlikely to have altered our main conclusions. However, the shape of the funnel plot seemed to be asymmetrical, suggesting that publication bias might have affected our findings.

Several limitations should be considered when interpreting the results of this study. First, most studies to date were conducted on homogenous Caucasian populations from Europe or the United States. Studies among racially and ethnically diverse cohorts, including Hispanics, African Americans and other ethnicities are needed.

Second, because the information used in the current research was based on data from observational studies, the characteristics of each study population and the different methodologies of these studies should be taken into account when interpreting the results of our analysis. For example, different inclusion criteria for selection of the 
participants might have influenced the results of this research. In the current study, four studies only concerned adults $(14,15,17,18)$, four studies $(14,15,17,18)$ considered the proportion of females, while the remaining studies (16) did not take into account age or gender ratios.

Differences in age distribution and gender ratio could also be potential causes of variation in the results. Additionally, although we tried to maximize our efforts to identify all relevant published studies in peer-reviewed journals, it is possible that some escaped our attention.

Third, NAFLD and HBV infection were included as other control groups. Data on body mass index and diabetes were not included in this analysis due to a lack of association with the presence of $\mathrm{CHC}$. Although false HCV positivity related to the PNPLA3 genotype was considered, there have been no reports of this association in the literature. Control group selection may affect the findings and interpretation of this research, and the use of different control groups could produce inconsistent results. The exclusion of NAFLD patients as controls in the current study precluded analysis of the contribution of steatosis independent of PNPLA3 status. Further meta-analysis on the NAFLD patient control subgroup with a larger sample size will be required in the future. It is assumed that a combined analysis based on studies with large samples can provide more accurate conclusions.

\section{Conclusions}

In conclusion, our analysis showed an association between gene polymorphisms of PNPLA3 and the presence of CHC; at least in Caucasians. This association had indications of possible publication bias, some heterogeneity, and less pronounced associations in prospective studies than in retrospective studies. Homogeneity of the methods for evaluating the degree of $\mathrm{CHC}$, common gender, age and ethnicity would be critical to confirm the absence of association and, therefore, the lack of a causal role of gene polymorphisms of PNPLA3 in patients with CHC. Furthermore, the probable association of PNPLA3 polymorphism with the natural history of hepatitis $C$, such as spontaneous clearance of HCV acute infection, maybe another hypothesis for explanation of the difference in distribution of PNPLA3 genotypes in CHC and the control group. Given the importance of this issue, further prospective rigorous studies are required to confirm our results and to evaluate the potential of PNPLA3 to serve both as a predictor and a therapeutic target in $\mathrm{CHC}$.

\section{Acknowledgments}

The authors thank all the contributors, who provided help and assistance.

\section{Footnote}

Authors' Contribution:Study concept and design: Haiying Zhang, Yongning Xin and Shiying Xuan. Acquisition of data: Haiying Zhang and Li Xue. Analysis and interpretation of data: Haiying Zhang, Li Xue and Lizhen Chen. Drafting of the manuscript: Haiying Zhang and Shunshun Jiang. Critical revision of the manuscript for important intellectual content: Haiying Zhang and Shunshun Jiang. Statistical analysis: Haiying Zhang and Lizhen Chen. Administrative, technical, and material support: Yongning Xin and Shiying Xuan. Study supervision: Yongning Xin and Shiying Xuan.

\section{References}

1. Khodabandehloo M, Roshani D. Prevalence of hepatitis C virus genotypes in Iranian patients: a systematic review and meta-analysis. Hepat Mon. 2014;14(12):e22915. doi: 10.5812/hepatmon.22915. [PubMed: 25685164]

2. Seeff LB. The history of the "natural history" of hepatitis C (1968-2009). Liver Int. 2009;29 Suppl 1:89-99. doi: 10.1111/j.14783231.2008.01927.x. [PubMed:19207971]

3. Urabe Y, Ochi H, Kato N, Kumar V, Takahashi A, Muroyama R, et al. A genome-wide association study of HCV-induced liver cirrhosis in the Japanese population identifies novel susceptibility loci at the MHC region. J Hepatol. 2013;58(5):875-82. doi:10.1016/j. jhep.2012.12.024. [PubMed: 23321320]

4. Asselah T, Bieche I, Sabbagh A, Bedossa P, Moreau R, Valla D, et al. Gene expression and hepatitis C virus infection. Gut. 2009;58(6):846-58. doi: 10.1136/gut.2008.166348. [PubMed: 19074178]

5. Romeo S, Kozlitina J, Xing C, Pertsemlidis A, Cox D, Pennacchio LA, et al. Genetic variation in PNPLA3 confers susceptibility to nonalcoholic fatty liver disease. Nat Genet. 2008;40(12):1461-5. doi:10.1038/ng.257. [PubMed:18820647]

6. Ke PY, Chen SS. Hepatitis C virus and cellular stress response: implications to molecular pathogenesis of liver diseases. Viruses. 2012;4(10):2251-90. doi:10.3390/v4102251. [PubMed: 23202463]

7. Everhart JE, Lok AS, Kim HY, Morgan TR, Lindsay KL, Chung RT, et al. Weight-related effects on disease progression in the hepatitis $\mathrm{C}$ antiviral long-term treatment against cirrhosis trial. Gastroenterology. 2009;137(2):549-57. doi: 10.1053/j.gastro.2009.05.007. [PubMed:19445938]

8. Dongiovanni P, Donati B, Fares R, Lombardi R, Mancina RM, Romeo S, et al. PNPLA3 I148M polymorphism and progressive liver disease. World J Gastroenterol. 2013;19(41):6969-78. doi: 10.3748/ wjg.v19.i41.6969. [PubMed: 24222941]

9. Singal AG, Manjunath H, Yopp AC, Beg MS, Marrero JA, Gopal P, et al. The effect of PNPLA3 on fibrosis progression and development of hepatocellular carcinoma: a meta-analysis. Am J Gastroenterol. 2014;109(3):325-34. doi: 10.1038/ajg.2013.476. [PubMed: 24445574]

10. Zampino R, Florio A, Coppola N, Cirillo G, Macera M, Marrone A, et al. PNPLA3 I148M variant as a risk factor for carotid atherosclerosis in chronic hepatitis C. Int J Cardiol. 2014;172(1):291-2. doi: 10.1016/j.ijcard.2013.12.231. [PubMed: 24461483]

11. Sookoian S, Gianotti TF, Gonzalez CD, Pirola CJ. Association of the C-344T aldosterone synthase gene variant with essential hypertension: a meta-analysis. J Hypertens. 2007;25(1):5-13. doi: 10.1097/01.hjh.0000254372.88488.a9. [PubMed:17143166]

12. Conn VS, Rantz MJ. Research methods: managing primary study quality in meta-analyses. Res Nurs Health. 2003;26(4):322-33. doi: 10.1002/nur.10092. [PubMed:12884420]

13. Huwiler-Muntener K, Juni P, Junker C, Egger M. Quality of reporting of randomized trials as a measure of methodologic quality. JAMA. 2002;287(21):2801-4. [PubMed: 12038917]

14. Nischalke HD, Berger C, Luda C, Berg T, Muller T, Grunhage F, et al. The PNPLA3 rs738409148M/M genotype is a risk factor for liver cancer in alcoholic cirrhosis but shows no or weak association in hepatitis C cirrhosis. PLoS One. 2011;6(11):e27087. doi: 10.1371/ journal.pone.0027087. [PubMed: 22087248]

15. Valenti L, Rumi M, Galmozzi E, Aghemo A, Del Menico B, De Nicola S, et al. Patatin-like phospholipase domain-containing 3 I148M polymorphism, steatosis, and liver damage in chronic hepatitis 
C. Hepatology. 2011;53(3):791-9. doi: 10.1002/hep.24123. [PubMed: 21319195]

16. Miyashita M, Ito T, Sakaki M, Kajiwara A, Nozawa H, Hiroishi K, et al. Genetic polymorphism in cyclooxygenase-2 promoter affects hepatic inflammation and fibrosis in patients with chronic hepatitis C. J Viral Hepat. 2012;19(9):608-14. doi: 10.1111/j.13652893.2011.01580.x. [PubMed: 22863264]

17. Valenti L, Rametta R, Ruscica M, Dongiovanni P, Steffani L, Motta $\mathrm{BM}$, et al. The I148M PNPLA3 polymorphism influences serum adiponectin in patients with fatty liver and healthy controls. BMC Gastroenterol. 2012;12:111. doi: 10.1186/1471-230X-12-111. [PubMed: 22898488]

18. Ezzikouri S, Alaoui R, Tazi S, Nadir S, Elmdaghri N, Pineau P, et al. The adiponutrin I148M variant is a risk factor for HCVassociated liver cancer in North-African patients. Infect Genet Evol. 2014;21:179-83. doi: 10.1016/j.meegid.2013.11.005. [PubMed: 24269995]

19. DerSimonian R, Laird N. Meta-analysis in clinical trials. Controlled Clinical Trials. 1986;7(3):177-88. doi:10.1016/0197-2456(86)90046-2. [PubMed: 3802833]

20. Egger M, Davey Smith G, Schneider M, Minder C. Bias in meta-analysis detected by a simple, graphical test. BMJ.1997;315(7109):62934. [PubMed: 9310563]

21. He S, McPhaul C, Li JZ, Garuti R, Kinch L, Grishin NV, et al. A sequence variation (I148M) in PNPLA3 associated with nonalcoholic fatty liver disease disrupts triglyceride hydrolysis.J Biol Chem. 2010;285(9):6706-15. doi: 10.1074/jbc.M109.064501. [PubMed: 20034933]

22. Huang Y, He S, Li JZ, Seo YK, Osborne TF, Cohen JC, et al. A feed-forward loop amplifies nutritional regulation of PNPLA3. Proc Nat Acad Sci U S A. 2010;107(17):7892-7. doi: 10.1073/pnas.1003585107. [PubMed: 20385813]

23. Pirazzi C, Adiels M, Burza MA, Mancina RM, Levin M, Stahlman $\mathrm{M}$, et al. Patatin-like phospholipase domain-containing
3 (PNPLA3) I148M (rs738409) affects hepatic VLDL secretion in humans and in vitro. J Hepatol. 2012;57(6):1276-82. doi: 10.1016/j. jhep.2012.07.030. [PubMed: 22878467]

24. Kumari M, Schoiswohl G, Chitraju C, Paar M, Cornaciu I, Rangrez AY, et al. Adiponutrin functions as a nutritionally regulated lysophosphatidic acid acyltransferase. Cell Metab. 2012;15(5):691-702. doi:10.1016/j.cmet.2012.04.008. [PubMed:22560221]

25. Trepo E, Pradat P, Potthoff A, Momozawa Y, Quertinmont E, Gustot $\mathrm{T}$, et al. Impact of patatin-like phospholipase-3 (rs738409 C>G) polymorphism on fibrosis progression and steatosis in chronic hepatitis C. Hepatology. 2011;54(1):60-9. doi: 10.1002/hep.24350. [PubMed: 21488075]

26. Corradini SG, Burza MA, Molinaro A, Romeo S. Patatin-like phospholipase domain containing 3 sequence variant and hepatocellular carcinoma. Hepatology. 2011;53(5):1776. doi: 10.1002/ hep.24244. [PubMed: 21351112]

27. Leandro G, Mangia A, Hui J, Fabris P, Rubbia-Brandt L, Colloredo $\mathrm{G}$, et al. Relationship between steatosis, inflammation, and fibrosis in chronic hepatitis C: a meta-analysis of individual patient data. Gastroenterology. 2006;130(6):1636-42. doi: 10.1053/j.gastro.2006.03.014. [PubMed:16697727]

28. Kau A, Vermehren J, Sarrazin C. Treatment predictors of a sustained virologic response in hepatitis B and C. J Hepatol. 2008;49(4):634-51. doi: 10.1016/j.jhep.2008.07.013. [PubMed: 18715665]

29. Walsh MJ, Vanags DM, Clouston AD, Richardson MM, Purdie DM, Jonsson JR, et al. Steatosis and liver cell apoptosis in chronic hepatitis C: a mechanism for increased liver injury. Hepatology. 2004;39(5):1230-8. doi:10.1002/hep.20179. [PubMed:15122751]

30. Sterne JA, Juni P, Schulz KF, Altman DG, Bartlett C, Egger M. Statistical methods for assessing the influence of study characteristics on treatment effects in 'meta-epidemiological' research. Stat Med.2002;21(11):1513-24. doi:10.1002/sim.1184. [PubMed:12111917] 